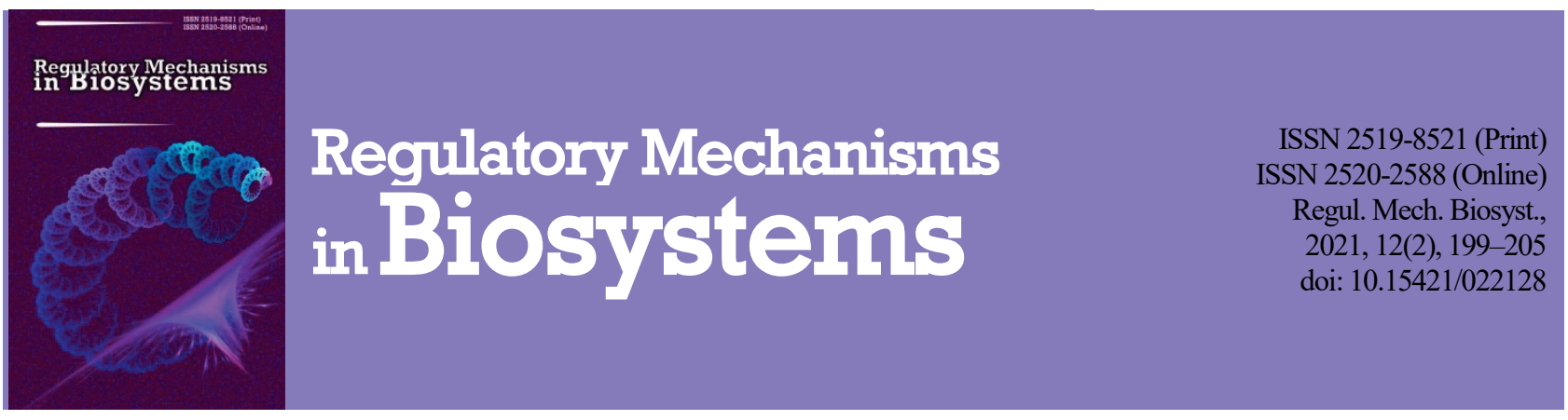

\title{
Monitoring of distribution of antibiotic-resistant strains of microorganisms in patients with dysbiosis of the urogenital tract
}

\author{
T. Sklyar*, V. Gavryliuk*, K. Lavrentieva*, N. Kurahina*, T. Lykholat*, K. Zaichenko*, M. Papiashvili**, \\ O. Lykholat***, D. Stepansky**** \\ * Oles Honchar Dnipro National University, Dnipro, Ukraine \\ **Independent Laboratory INVITRO LLC, Dnipro, Ukraine \\ ***University of Customs and Finance, Dnipro, Ukraine \\ ****Dnipropetrovsk Medical Academy of Health Ministry of Ukraine, Dnipro, Ukraine
}

Article info

Received 08.05.2021

Received in revised form 01.06 .2021

Accepted 02.06.202

Oles Honchar Dnipro National

University, Gagarina av., 72,

Dnipro, 49010, Ukraine.

Tel.: +38-050-940-00-98

E-mail:microviro@ukr.net

Independent Laboratory INVITRO

LLC, Sviatoslav Khorobryi st., 38,

Dnipro, 49000, Ukraine.

Tel: $+38-067-280-59-03$

E-mail:lena96dp@gmail.com

University of Customs and Finance, Volodymyr Vernadskyst., 2/4, Dnipro, 49000, Ukraine.

$\mathrm{Tel} \cdot \mathbf{\cdot}+38-066-038-68-92$

E-mail:lykholat2010@ukrnet

Dnipropetrovsk Medical Academy of Health Ministry of Ukraine, Volodymyr Vernadskyst, 9 ,

Dnipro, 49000, Ukraine.

Tel.: +38-097-978-49-77.

E-mail:dstepansky@gmail.com
Sklyar, T., Gavryliuk, V., Lavrentieva, K., Kurahina, N., Lykholat, T., Zaichenko, K., Papiashvili, M., Lykholat, O., \& Stepansky, D. (2021). Monitoring of distribution of antibiotic-resistant strains of microorganisms in patients with dysbiosis of the urogenital tract. Regulatory Mechanisms in Biosystems, 12(2), 199-205. doi:10.15421/022128

Currently, the problem of the development of resistance to drugs among microorganisms that colonize the urogenital system is becoming especially relevant due to broadly distributed dysbiotic conditions of the reproductive system of men and women. Therefore, there should be constant monitoring of the qualitative and quantitative composition of microbiota of the urogential tract and determination of the levels of antibiotic-resistance of strains of conditionally pathogenic microorganisms in the reproductive system of various layers of the population. We monitored 774,375 people of various age and sex - patients of the independent diagnostic laboratory INVITRO in the city Dnipro in 2017-2019. Among the examined people, 640,783 of the patients were diagnosed with the development of dysbiotic disorders, accounting for $82.7 \%$ of the total amount of the applications for medical help. According to the results of identification of the range of dysbiotic conditions of the urogenital system of patients of different ages and sexes, we determined the dominating role of facultative anaerobes in the development of dysbiotic impairments caused by colonizations by large numbers of conditionally-pathogenic microorganisms: in women, Gardnerella accounted for $86.1 \%$, Staphylococcus $-63.2 \%$, Streptococcus $-54.1 \%$, Candida $-69.3 \%$; in men, Streptococcus were found in $83.0 \%$, Staphylococcus $-79.4 \%$, Corynebacterium $-54.2 \%$ and Candida $-37.6 \%$ of the cases. Share of obligate anaerobes was also quite large: women were diagnosed with Prevotella in 59.7\%, Peptostreptococcus in 53.2\%, Fusobacterium in $45.4 \%$ of the cases cases; men were observed to have Peptostreptococcus $62.4 \%$, Clostridium in $54.3 \%$, Bacteroides in $32.5 \%$ of the cases. We determined high parameters of frequency of diagnosing antibiotic-resistant isolates of conditionally pathogenic microorganisms that circulate in the urogenital tract of patients with dysbiotic impairments, belonging to the following families: Mycoplasmataceae- $78.6 \%$, Enterobacteriaceae $-56.0 \%$ and genera - Staphylococcus $-76.1 \%$, Gardnerella $-24.3 \%$, Corynebacterium $-21.2 \%$. The research revealed increase in the frequency of detection of strains of urapathogenic bacteria resistant to the applied antibiotic preparations in 2018-2019 compared with the data of 2017: increases of 10.3\% and 6.4\% in representatives of family Mycoplasmataceae resistant to ciprofloxacin and ofloxacin respectively, $4.8 \%$ and $4.0 \%$ in Enterobacteriaceae resistant to chloramphenicol and ampicillin respectively, and $8.9 \%$ in the genus Staphylococcus resistant to vancomycin.

Keywords: monitoring; antibiotic resistance; microbiota; dysbiosis; urogenital tract.

\section{Introduction}

According to the modern views, the microbial landscape of the urogenital tract plays an important role in the development of disease conditions. The revolution in the methodological approaches allows an assessment of the complex interactions between microbiota of the urogenital system and the macroorganism. Moreover, at the moment, there is a significant potential for progress in discovering biomarkers and stratification of the development of diseases, and also to determine mechanisms that lie at the basis of dynamic balance of microbial groups involved in inflammatory processes of the urogenital tract (Neugent et al., 2020).

Many inflammatory processes are caused by the development of dysbiotic condition of the reproductive system, which is characterized by absence of specific symptoms of disease, predominance of an asymptomatic clinical picture and high probability of development of chronic forms and, as a result, malfunctioning of the reproductive function. Currently, there are evidences that indicate relationship between dysbiotic impairments in the female reproductive system and infertility (Bitew et al., 2017; Aragón et al., 2018). Dysbiotic disorders vary in distribution and severity, and depend on ecological conditions of the region, age structure, health condition and lifestyle of representatives of various layers of the population. The highest amount of deviations was recorded for inhabitants of large industrial cities (Graspeuntner et al., 2018). The relevance of this problem, particularly for the city of Dnipro, makes it necessary to study ways and causes of distribution of dysbioses and development of timely and adequate correction of the composition of microbiota of people with dysbiotic impairments.

Microorganisms that inhabit various biotopes are rarely represented by one species, most of them co-exist in complex polymicrobial associations, forming biofilms. The etiological structure of non-specific urogenital diseases is not an exception and is often represented by associations of microorganisms, which should be taken into account when selecting a complex of laboratory studies and targeted reasoned therapy (Kamińska \& Gajecka, 2017). Of especial relevance is the possibility to detect in good 
time inflammatory process using modern diagnostic studies, the purpose of which is prevention of development of infections, dysbiotic conditions and reproductive disorders. Also, the broad range of variability in parameters of morbidity with urogenital infections indicates frequent diagnostic errors when determining the etiological role of some uropathogenic microorganism or other.

Nowadays, an especially relevant problem is the development of resistance to medical preparations among conditionally pathogenic microorganisms of the urogenital system (Ho et al., 2019). The data of the network of the European system of epidemiological surveillance of resistance to anti-microbial drugs indicate significant geographic differences in distribution of resistance to various classes of antibiotics. Resistant pathogen strains of infections were reported to be the causes of diseases in the South of Europe more often compared with the North of Europe (Machowska \& Lundborg, 2019). A subject of special concern is the rapid distribution of bacteria with multi-drug resistance, for the therapy of infectious diseases using antibiotics in each ecotope promotes development of resistant strains that become the reservoir for resistant genes (Palchykov et al., 2019; Zazharskyi et al., 2019, 2020; Rigo \& Tasca, 2020). These determinants are distributed by microorganisms and transferred from one biotope to another (Kudinova et al., 2019). Pathogenic bacteria, which every year are being increasingly isolated from the urogenital tract, display antibiotic resistance not only to classic antibacterial drugs, but the new ones as well. Such a boom in resistance is mainly associated with irrational antibiotic therapy. It is obviously necessary to raise awareness of the use of antibiotics and causes of the development of resistance among microorganisms (Boboia et al., 2020). From the microbiological perspective, the problem of antibiotic resistance is caused by losses of active groups of antimicrobial drugs, activity of which inevitably decreases as a result of development of resistance to them and insufficient level of microbiological diagnostics of antibiotic resistant microorganism strains (Burnham et al., 2017; Mediati et al., 2021).

Therefore, it is necessary to continuously monitor the distribution of antibiotic-resistant variants of microorganisms to provide effective prevention and treatment of infectious diseases taking into account the most probable pathogens and regional data on their sensitivity to antimicrobial drugs (Knight et al., 2019; Wagenlehner, 2020).

Thus, the objective of the study was determining the frequency of occurrence of antibiotic resistant strains of conditionally pathogenic microorganisms that colonize the urogenital tract of patients with dysbiotic impairments.

\section{Materials and methods}

To perform monitoring of circulation of antibiotic resistant strains of conditionally pathogenic microorganisms in the reproductive system, we examined 774,375 people of various age and sex - patients of the INVITRO Independent Diagnostic Laboratory in city Dnipro in the period of 2017-2019. All the monitored patients had signed an informed consent, and the study was conducted according to the Declaration of Helsinki Global Medical Association, the Statute of the Ukraine Association of Bioethics, normative positions about the ethics of the Ministry of the Healthcare of Ukraine No. 66 of 13.02.2006. The research involved the following anamnesis data: patients' age, sex, social status.

The composition of microbiome of the uragenital tract was studied using the method of polymerase chain reaction (PCR) employing amplifier DT-96/DT-322 (DNA-Technology LLC, Russia) in real time regime (RT PCR) using Androflor (DNA-Technology LLC, Russia) during the study of biomaterial from men and Femoflor 16 (DNA-Technology LLC, Russia) while studying biomaterial from women.

Clinical isolates of Mycoplasmataceae were identified using the following test systems: Mycoplasma - IST 2 (BioMerieux, France), Enterobacteriaceae - API 20E (BioMerieux, France), Staphylococcus - API Staph (BioMerieux, France), Streptococcus and Gardnerella - API 20 Strep (BioMerieux, France), Candida - API Candida (BioMerieux, France), Corynebacterium - API Coryne (BioMerieux, France), anaerobes - API 20 A (BioMerieux, France).

The extent of resistance of isolated strains of conditionally pathogenic bacteria to antibiotic preparations was determined using the disk diffusion method. Susceptibility of microorganisms to antibiotics was determined according to criteria CLSI (CLSI, 2018).

\section{Results}

Patients with suspicions of dysbiosis in microbiota of the urogenital tract were those who asked for help at the laboratory following doctors' recommendations, underwent planned medical examination, or came on their own will. Among them, 640,783 people were diagnosed with dysbiotic malfunctions, accounting for $82.7 \%$ of the all patients' complaints (Fig. 1).

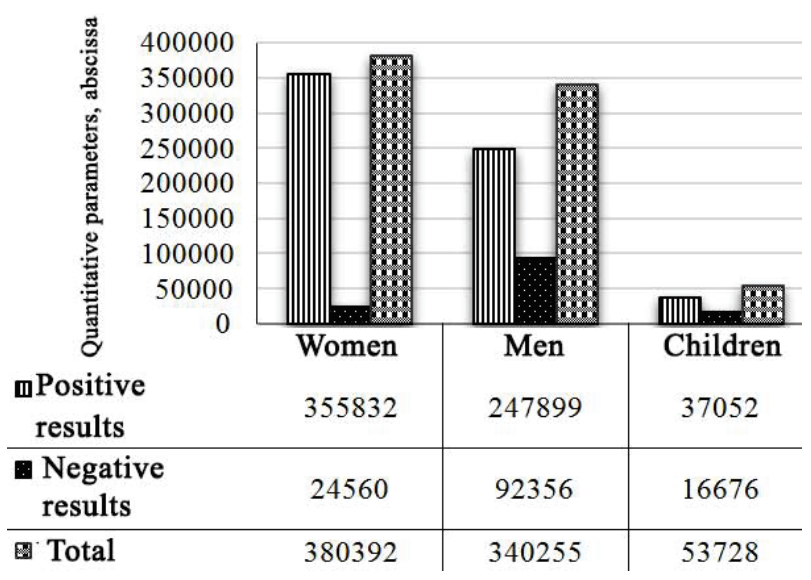

Fig. 1. Frequency of detection of dysbiotic malfunctions of the urogenital tract in the patients of various layers of the population for 2017-2019

Analysis of the structure of the contingent of patients examined according to sex allowed us to determine that the frequency of detection of positive results for women accounted for $93.5 \%$ of the number of complaints; and was $72.9 \%$ for men, and $69.0 \%$ for children.

As a result of the study of the anamnesis data, we analyzed the frequency of occurrence of dysbiosis in the urogenital tract of women of different age and status (Fig. 2). The greatest amount of dysbiotic disorders was found in the age groups of reproductive age: $25-30$ years $(41.2 \%$ of all the infected) and 15-25 years (32.3\%), among which $67.0 \%$ and $74.3 \%$, respectively were not married. Somewhat less frequency of detection of imbalances was recorded for women aged $30-40$ years (17.2\% of all dysbioses), $32.1 \%$ comprising unmarried ones. The lowest percentage of disorders in microbiocenosis of the urogenital system was recorded in people aged 40-60 years $(6.7 \%)$ and older than $60(2.6 \%)$, among which the unmarried ones accounted for $12.0 \%$ and $5.0 \%$ correspondingly.

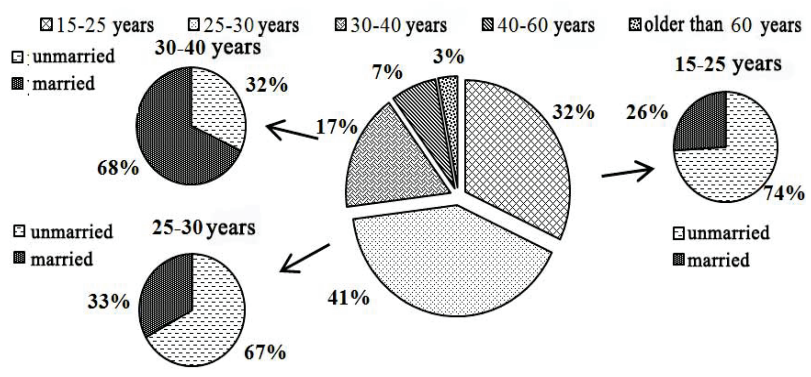

Fig. 2. Frequency of detection of dysbioses in the urogenital system of women of different age and social status

In men, the development of dysbiosis was most often observed at the age of 25 to 30 years ( $50.2 \%$ of all the patients) and 15 to 25 years $(35.1 \%$ of the cases), out of which $41.2 \%$ and $78.3 \%$ of the patients, respectively, were unmarried (Fig. 3). The lowest frequency of the complaints and positive results for dysbiotic malfunctions in the microbiome of the urogenital tract was found among men of the older generations: $40-60$ and older than 60 (of which $17.4 \%$ and $2.6 \%$, respectively, were unmarried).

According to the results of the microbiological examination, we determined the frequency of occurrence of facultative and obligate anaerobic bacteria in the urogenital tract of men and women, as well as the domina- 
ting role of facultative anaerobes in the development of dysbiotic malfunctions (Table 1).

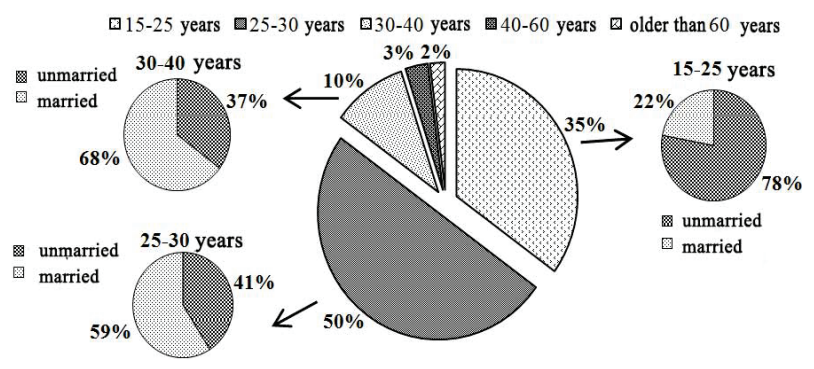

Fig. 3. Frequency of detection of dysbioses of the urogenital system of men of different age and social status

In the urogenital tract of girls from birth to 14 years, an insignificant amount of strains of conditionally pathogenic microorganisms was deter- mined, among which the prevailing number comprised representatives of Peptostreptococcus genus - 68.1\%, and also: Gardnerella - 23.4\%, Mycoplasma $-20.2 \%$ and Escherichia - $18.3 \%$ of cases; lower parameters of occurrence were recorded for strains of yeast-like fungi Candida $-13.0 \%$ and bacteria of Ureaplasma - 12.2\%. In small amount, we detected facultative and obligate-anaerobic bacteria of the following genera: Corynebacterium - in $9.4 \%$ of the cases, Bacteroides - 4.0\%, Fusobacterium $5.2 \%$, Mobiluncus - $3.2 \%$, Propionibacterium - in $4.0 \%$ and the Enterobacteriaceae family $-7.4 \%$ of the cases.

Analysis of clinical samples from women of reproductive age (18-35 years) allowed us to determine a microbial landscape of another pattern. Therefore, high frequency was seen for facultative-anaerobic microorganisms of the following genera: Gardnerella $-86.1 \%$, Staphylococcus $-63.2 \%$, Steptococcus - 54.1\%, Mycoplasma - 15.4\%, representatives of the Enterobacteriaceae family $-26.0 \%$. Percentage of the content of fungi of Candida genus accounted for $69.3 \%$ of the overall amount of isolates.

\section{Table 1}

Comparative analysis of the composition of the urogenital system in patients of different sex with dysbiotic disorders

\begin{tabular}{|c|c|c|c|c|c|c|}
\hline \multirow[b]{2}{*}{$\begin{array}{c}\text { Species } \\
\text { of microorganisms }\end{array}$} & \multicolumn{3}{|c|}{ Women } & \multicolumn{3}{|c|}{ Men } \\
\hline & $\begin{array}{c}\text { norm, } \\
\text { CFU/mL }\end{array}$ & $\begin{array}{l}\text { dysbiosis, } \\
\text { CFU/mL }\end{array}$ & $\begin{array}{c}\text { frequency of detection of } \\
\text { dysbiosis, } \%\end{array}$ & $\begin{array}{c}\text { norm, } \\
\text { CFU } / \mathrm{mL}\end{array}$ & $\begin{array}{l}\text { dysbiosis, } \\
\text { CFU/mL }\end{array}$ & $\begin{array}{c}\text { frequency of diagnosing } \\
\text { dysbiosis, } \%\end{array}$ \\
\hline \multicolumn{7}{|c|}{ Facultative anaerobic bacteria } \\
\hline Lactobacillus spp. & $10^{7}-10^{9}$ & $\leq 10^{7}$ & 57.0 & $10^{6}$ & - & - \\
\hline Staphylococcus spp. & $10^{3}-10^{4}$ & $\geq 10^{4}$ & 63.2 & $10^{4}$ & $\geq 10^{4}$ & 79.4 \\
\hline Corynebacterium spp. & $10^{4}-10^{5}$ & $\geq 10^{5}$ & 37.3 & $10^{4}$ & $\geq 10^{4}$ & 54.2 \\
\hline Steptococcus spp. & $10^{4}-10^{5}$ & $\geq 10^{5}$ & 54.1 & $10^{4}$ & $\geq 10^{4}$ & 83.0 \\
\hline Gardnerella spp. & $10^{6}$ & $>10^{6}$ & 86.1 & - & + & 11.3 \\
\hline Enterobacteriaceae & $10^{3}-10^{4}$ & $\geq 10^{4}$ & 26.0 & - & + & 5.6 \\
\hline Ureaplasma spp. & $10^{3}$ & $\geq 10^{3}$ & 7.0 & _- & + & 19.2 \\
\hline Mycoplasma spp. & $10^{3}$ & $\geq 10^{3}$ & 15.4 & - & + & 14.4 \\
\hline \multicolumn{7}{|c|}{ Obligate anaerobic bacteria } \\
\hline Peptostreptococcus spp. & $10^{3}-10^{4}$ & $\geq 10^{4}$ & 53.2 & - & + & 62.4 \\
\hline Lactobacillus spp. & $10^{7}-10^{9}$ & $<10^{7}$ & 54.1 & $10^{6}$ & & 14.3 \\
\hline Eubacterium spp. & $<10^{3}$ & $\geq 10^{3}$ & 16.0 & - & + & 3.2 \\
\hline Bacteroides spp. & $10^{3}-10^{4}$ & $\geq 10^{4}$ & 9.3 & _- & + & 32.5 \\
\hline Fusobacterium spp. & $<10^{3}$ & $\geq 10^{3}$ & 45.4 & _- & + & 13.3 \\
\hline Veillonella spp. & $<10^{3}$ & $\geq 10^{3}$ & 4.2 & _- & + & - \\
\hline Propionibacterium spp. & $<10^{4}$ & $\geq 10^{4}$ & 24.0 & - & + & 6.2 \\
\hline Bifidobacterium spp. & $10^{3}-10^{7}$ & $\leq 10^{3}$ & 67.2 & $10^{5}$ & - & 7.4 \\
\hline Clostridium spp. & $<10^{4}$ & $\geq 10^{4}$ & 27.1 & - & + & 54.3 \\
\hline Prevotella spp. & $<10^{4}$ & $\geq 10^{4}$ & 59.7 & - & + & 3.4 \\
\hline Porphyromonas spp. & $<10^{3}$ & $\geq 10^{3}$ & 26.8 & - & + & 16.4 \\
\hline Mobiluncus spp. & $<10^{4}$ & $\geq 10^{4}$ & 21.2 & - & + & 9.3 \\
\hline \multicolumn{7}{|c|}{ Fungi } \\
\hline Candida spp. & $\geq 10^{4}$ & $\geq 10^{4}$ & 69.3 & - & + & 37.6 \\
\hline
\end{tabular}

Among dysbioses, obligate anaerobes of the following genera occurred somewhat more frequently: Prevotella $-59.7 \%$, Peptostreptococcus $-53.2 \%$, Fusobacterium - 45.4\%, Clostridium - 27.1\%, Propionibacterium - 24.0\%, Porphyromonas - 26.8\%, Mobiluncus - 21.2\%; and at the same time, Lactobacillus and Bifidobacterium were present, but were somewhat less numerous $-10^{1}-10^{7} \mathrm{CFU} / \mathrm{mL}$. Similarly to dysbiotic conditions of the reproductive system of women, the development of dysbiosis in most men was also due to conditionally pathogenic microorganisms that colonize the reproductive organs ( $76 \%$ of cases). Therefore, most often $\left(10^{5}-10^{8} \mathrm{CFU} / \mathrm{mL}\right)$, there were found facultative anaerobes: Steptococcus in $83.0 \%$, Staphylococcus - 79\%, Corynebacterium - 54\% of the cases; and as for the presence of other representatives of this group, the fact of dysbiotic malfunctioning was recorded even when they were detected in small amounts: Candida -37\%, Mycoplasma-14\%, Gardnerella $-11 \%$, Enterobacteriaceae $-5 \%$ of the cases.

Presence of obligate anaerobes, other than anaerobic strains of Lactobacillus $\left(10^{6} \mathrm{CFU} / \mathrm{mL}\right)$ and Bifidobacterium $\left(10^{5} \mathrm{CFU} / \mathrm{mL}\right)$, is also an evidence of dysbiotic condition of the reproductive tract of men. As a result of the research, $62.4 \%$ of patients were determined to have large amounts $\left(10^{2}-10^{10} \mathrm{CFU} / \mathrm{mL}\right)$ of bacteria of genera Peptostreptococcus, $54.3 \%$ - Clostridium, 32.5\% - Bacteroides, $16.4 \%$ - Porphyromonas, $13.3 \%$ - Fusobacterium genera. At the next stage, we carried out a study to determining resistance of the isolated cultures of conditionally pathogenic microorganisms, pathogens of dysbacterioses of the urogenital tract, to antibiotic drugs. Therefore, it is important to assess annual dynamics of changes in sensitivity of microorganisms based on continuous research of these properties in all etiologically significant strains isolated from the clinical material.

During the period of 2017-2019, 420,014 isolates of bacteria of the Staphylococcus genus were isolated, of which 39,319 (76\%) demonstrated resistance to vancomycin, which belongs to the group of drugs of "strategic reserve", and resistance to the traditional drugs was displayed by erythromycin and chloramphenicol-59,222 (14.1\%) and 60,062 (14.3\%) respectively. The analysis of the parameters of frequency of detecting resistant strains of Staphylococcus in 2018-2019, compared with 2017, revealed their significant increase in resistance to active antibiotics: $0.7 \%$ to erythromycin, $0.9 \%$ to chloramphenicol, $8.9 \%$ to vancomycin (Fig. 4).

Among 397,905 clinical isolates of Streptococcus genus that colonized the reproductive organs of patients, 49,738 (12.5\%) were found resistant to clindamycin, 23,079 (5.8\%) - to levofloxacin, 23,078 (5.7\%) to levomycetin, $12,335(3.1 \%)$ - to ciprofloxacin. During the studies in 2018-2019, we determined that the amount of resistant strains of Streptococcus increased compared with the data of 2017: $0.3 \%$ to ciprofloxacin, $0.5 \%$ to levofloxacin, $0.3 \%$ to levomycetin, $0.5 \%$ to clindamycin (Fig. 5 ).

Results of the research of antibiotic-resistance in 104,911 clinical variants - representatives of the Enterobacteriaceae family revealed that $58,750(56 \%)$ exerted resistance to chloramphenicol, $44,063(42 \%)$ - to ampicillin, 32,522 (31\%) - to doxycycline (Fig. 6). 


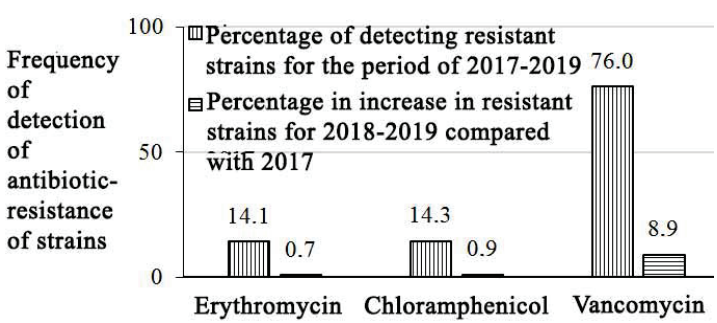

Fig. 4. Frequency of detection of antibiotic-resistant strains of bacteria of Staphylococcus genus

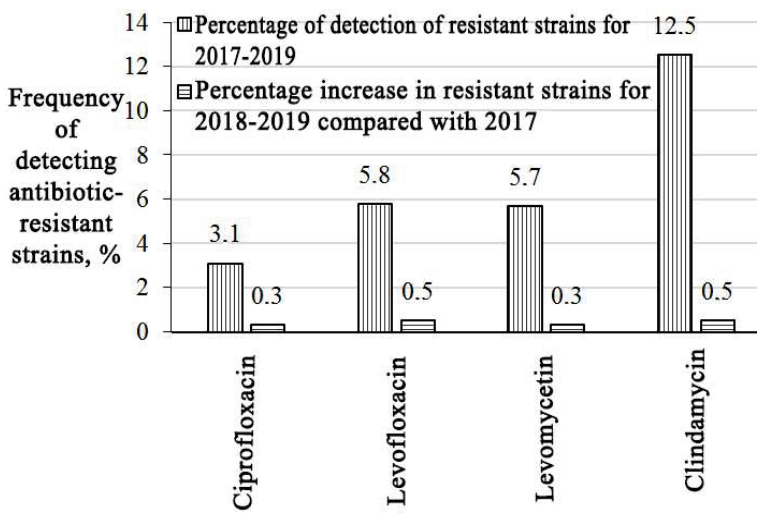

Fig. 5. Frequency of detection of antibiotic resistant strains of bacteria of Streptococcus genus

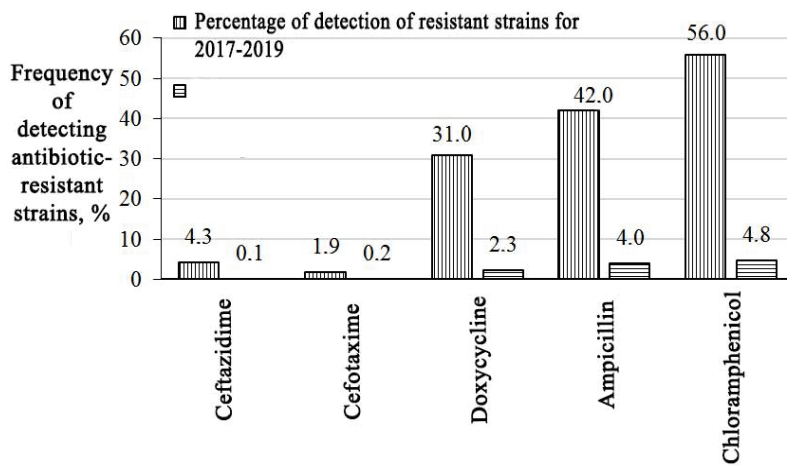

Fig. 6. Frequency of detection of antibiotic-resistant strains of bacteria of Enterobacteriaceae family

The number of isolates of intestinal bacteria that were resistant against antibiotic preparations also had a tendency to growth, as indicated in the last two years compared with the data of 2017: $0.1 \%$ increase against ceftazidime, $0.2 \%$ to cefotaxime, $2.3 \%$ to doxycycline, $4.0 \%$ to ampicillin, $4.8 \%$ against chloramphenicol.

For Gardnerella, there was also seen the tendency toward increase in the frequency of occurrence of resistant variants during the last two years of the research compared with the data of 2017: 1.2\% against tetracycline, $4.7 \%$ to ampicillin, $3.1 \%$ to erythromycin, $4.8 \%$ to ofloxacin (Fig. 7 ).

From the reproductive tract of the patients, there were isolated 265,524 isolates of bacteria of the Corynebacterium genus. The most frequently detected resistant variants were those against rifampicin $55,760(21 \%)$, clarithromycin - 45,139 (17\%), penicillin - 42,484 (16\%), erythromycin - 38,501 (14.5\%), azithromycin - 29,208 (11\%). Increase in the number of resistant cultures for the two recent years was: $1.6 \%$ to azithromycin, $1.8 \%$ to erythromycin, $1.8 \%$ to penicillin, $2.0 \%$ to clarithromycin, $2.5 \%$ to rifampicin (Fig. 8).

Among 160,090 isolates of Mycoplasmataceae family, 125,831 (78.6\%) strains were resistant to ciprofloxacin, 78,444 (49\%) to ofloxacin, $12,807(8 \%)$ to azithromycin, $6,404(4 \%)$ to doxycycline, $3,362(2.1 \%)$ to josamycin (Fig. 9). Comparing the parameters of resistance for the researched period, we determined that frequency of detection of antibioticresistant variants increased by $10.3 \%$ to ciprofloxacin, $6.4 \%$ - to ofloxacin, $0.9 \%$ - to azithromycin.

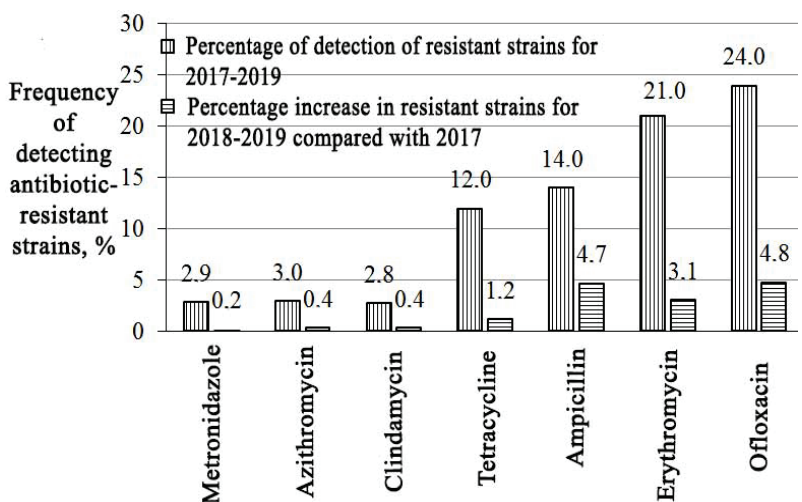

Fig. 7. Frequency of detection of antibiotic-resistant strains of bacteria of Gardnerella genus

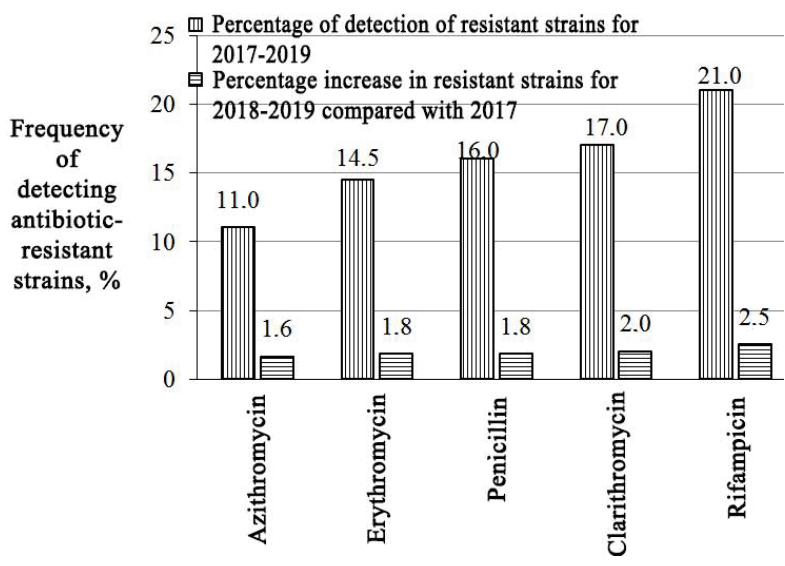

Fig. 8. Frequency of detection of antibiotic-resistant strains of bacteria of Corynebacterium genus

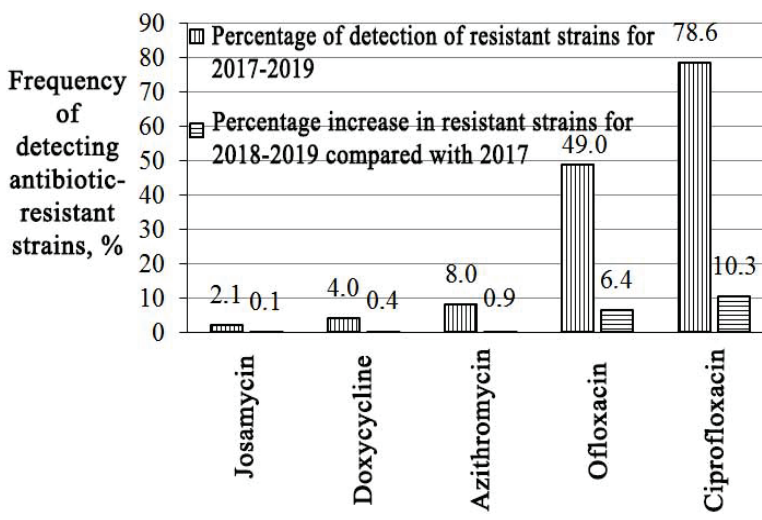

Fig. 9. Frequency of detecting antibiotic-resistant strains of bacteria of Mycoplasmataceae family

\section{Discussion}

Inflammatory diseases of the urogenital organs are still a relevant medical problem due to the high level of morbidity, chronic course, insufficiently effective therapy leaving possibilities of development of recurring diseases or residual symptoms of infection. Biota of the urogenital system is characterized by individual populations of microbiomes, caused by variety of interrelations of vegetating microorganisms, their antagonism and synergism. Normal growth of the urogenital biotope is continuous, but in non-favourable conditions, there occurs a shift in the components of microbiota, followed by rapid decrease in concentration of the resident microbiome, and the number of facultative-anaerobic and aerobic microorganisms tends to increase. Ecological imbalances in this biotope cause decrease in colonization resistance of the mucous membranes, which in turn leads to formation of new microbial associations that may mutually 
increase their pathogenic properties. Over the last 20-30 years, there has been an increase in the number of different pathologic conditions based on impaired normal microbiocoenosis of the human organism - dysbacteriosis. In such a condition, the organism is provided with less vitamins and microelements, various types of metabolism suffer, developing conditions for reduction of the immunity and development of diseases of the reproductive organs of men and women. The main causes of dysbacteriosis may be considered to be uncontrolled ingression of and inadequate treatment with antibiotics, stressful situations, climate changes, overcooling, failure to follow the hygiene norms, chronic fatigue, any inflammations of the organs of the lesser pelvis, caused by bacteria and viruses, and also dysbacteriosis of the intestine (Agar et al., 2020). Most often, disorders in the normocoenosis of the reproductive organs of a macroorganism leads to sexually transmitted diseases, which in turn lead to complicated consequences such as deterioration of life quality, reduction of immunity and development of inflammatory processes, pathological course of pregnancy, threat of miscarriage, rupture of the fetal membranes, premature birth, male and female infertility (Schuppe et al., 2017).

According to the analyzed data, the frequency of detection of positive results for women exceeded this parameter for men and children. This may be explained by the fact that microbiota of the female urogenital tract is more diverse and variable than in men and children, especially in the first year of life and in puberty, when it is developing and is subject to the impact of different factors (MacIntyre et al., 2017; Abelson et al., 2018).

Studies of microbiota of the urogenital tract of women are related to certain difficulties, such as the fact that the lower sections of the reproductive organs in the norm contain a diverse microbiome that is subject to changes in certain age periods of life of the macroorganism (Koedooder et al., 2019). The vagina of newborns is inhabited by lactic bacteria, but they are gradually replaced with cocci group (mostly Staphylococcus epidermidis), which remain characteristic up to the period of sexual maturation. During menopause, in the reproductive tract of females, the microorganisms of the cocci group become dominant again (Muhleisen \& HerbstKralovetz, 2016; Li \& Ma, 2019). At the reproductive age, the dominant bacteria in the content of microbiome are aerobic lactic bacteria in association with other saprophytes. There occur representatives of genera Lactobacterium, Peptococcus, Peptostreptococcus, Corynebacterium, Staphylococcus (Martin \& Mazzarro, 2016; Xu et al., 2020).

According to our research, in women, facultative anaerobes were determined to play the dominant role in the development of dysbiotic malfunctions caused by colonization by conditionally-pathogenic microorganisms in large amounts (Gardnerella, Staphylococcus, Streptococcus, Candida) and also obligate anaerobes (Prevotella, Peptostreptococcus, Fusobacterium). The reasons of such inhabitation of the vagina by conditionally pathogenic microorganisms in the examined women of reproductive age may be high frequency of sexual activity, interruption of pregnancy, irrational consumption of broad-spectrum antibiotics, oral and invasive contraceptives, steroid hormones (Ceccarani et al., 2019). The said factors inhibit colonizing resistance of beneficial microorganisms and massive settlement of obligate and facultative-anaerobic microbiota of the urogenital tract occurs, causing dysbioses of various degrees of severity (Song et al., 2020; Stout et al., 2020).

In male reproductive organs, a relatively low temperature is maintained, which does not allow the bacteria present in them to intensely reproduce. Therefore, dysbiosis of the urogenital tract of men occurs more rarely than in women (Lewis et al., 2017; Bajic et al., 2019). At the same time, dysbiotic disorders of the reproductive systems of men may occur at the initial stage in the urethra, and then be distributed to other organs of the prostate-vesicular complex with formation of the inflammatory process in one or several loci at the same time (Oztekin et al., 2018). It was also found that coagulase-negative staphylococci, streptococci, corynebacteria at titer of up to $10^{4} \mathrm{CFU} / \mathrm{mL}$ and also lactobacilli and bifidobacteria in the norm may vegetate in the reproductive organs of healthy men, but if the titers of facultative-anaerobic microorganisms exceed the parameters of the norm, even without clearly noticeable symptomatics, it is an evidence of dysbiosis of the urogenital tract of men (Tang, 2017). Malfunctions in microbiocoenosis of men is often a consequence of disorder in the microbiome of the vaginas of their sexual partners, which in such conditions explains the prevalence of the representatives of facultative (genera Strep- tococcus, Staphylococcus, Corynebacterium, Candida) and obligate anaerobes (genera Peptostreptococcus, Clostridium, Bacteroides) in the urogenital tract.

Furthermore, a quite common reason for changes in the quantitative and qualitative compositions of microbiocoenosis in the male reproductive system is development of chronic diseases of metabolic and infectious natures: diabetes, kidney stone disease, phimosis, sexually transmitted infections, and also violations of sanitary-hygienic norms, etc. (Li \& Ma, 2019; Lee et al., 2020). In cases of inadequate treatment or self-treatment, a tendency towards emergence of chronic prostatitis was observed (Shoskes et al., 2016). Most often, it develops against the background of decrease in the colony resistance caused by protective epithelial and immune mechanisms (Ortega Martell, 2020). First of all, the microbiocoenosis undergoes change as a result of deficiency or elimination of representatives of the normal microbiome that displays antagonism towards allochthonous bacteria or prevents their adhesion on epitheliocytes; secondly, there occurs deficiency of factors of local non-specific resistance. This results in development of favourable conditions for colonization of the urogenital tract by allochthonous microorganisms, i.e. dysbiosis takes place, characterized by deficiency or absence of representatives of normal microbiota or presence of conditionally pathogenic microorganisms with notably persistent properties (Magistro \& Stief, 2019).

The essential link in the therapy of infectious diseases caused by conditionally pathogenic microorganisms is prescription of antibacterial preparations. But in cases of associations of microorganisms during bacteriological inoculations, the question is what effective antibiotics to chose, because pathogens of inflammation may belong to different taxonomic groups and have differences in the susceptibility to antibacterial preparations. Over $70 \%$ of infections are caused by microorganisms resistant to several or a number of antibiotics (Bondarenko et al., 2017; Drago et al., 2020).

During the period of 2017-2019, there were determined high parameters of detection of antibiotic-resistant isolates of conditionally pathogenic microorganisms that circulate in the reproductive tract of patients with dysbiotic disorders. Therefore, $49.0-78.6 \%$ of the representatives of the Mycoplasmataceae family were resistant to drugs of the fluoroquinolone group; $56 \%$ of Enterobacteriaceae - to chloramphenicol, $42 \%$ to ampicillin, $31 \%$ to doxycycline; $76 \%$ of Staphylococcus genus - to vancomycin.

The data obtained confirm the results of a number of other researchers. According to the data of Karou et al. (2012), bacteria of Escherichia coli, Klebsiella pneumoniae and K. ozenae, isolated from the clinical samples from patients suffering dysbiotic malfunctions of the reproductive system, were characterized by the highest level of resistance to ampicillin respectively $60 \%, 72 \%$ and $50 \%$ of the strains, also $55 \%, 49 \%$ and $50 \%$ were resistant to amoxicillin/clavulanate, $63 \%, 50 \%$ and $30 \%$ to trimetaprim-sulfamethoxazole, $30 \%, 32 \%$ and $25 \%$ of the cultures to chloramphenicol. Saad et al. (2020) revealed that in the conditions of quantitative and qualitative changes in the composition of microbiota of the urogenital tract of men and women of reproductive age, more than $60 \%$ of all isolates of enterobacteria were characterized by resistance to $\beta$-lactam antibiotics and preparations of the tetracycline group; $40-60 \%$ of the cultures were resistant to fluoroquinolones and around $30 \%$ of strains - to chloramphenicol. Over $70 \%$ of the isolates of Staphylococcus aureus were resistant to penicillins (including methicillin), co-trimoxazole, clindamycin and nalidixic acid. We have to note the fact that $40 \%$ of the cultures of Staphylococcus aureus were resistant to vancomycin - drug that for a long time remained the agent of choice for treating infections caused by MRSA strains. However, in the mid 1990s, first in Japan and USA, and them in other countries, there began to emerge reports about the increasing frequency of isolating methicillin-resistant Staphylococcus with reduced susceptibility to vancomycin (Sirichoat et al., 2016; Tran \& Rybak, 2018).

As with Mycoplasma isolated from men and women with dysbioses of the urogenital tract, a number of scientists report their high level of resistance to macrolides (Karou et al., 2012; Conway et al., 2019). According to the data of Conway et al. (2019), in various countries around the world, frequency of detection of clinical strains of Mycoplasma resistant to macrolide antibiotics accounted for 30-77\%: in Finland and the Netherlands $-31 \%$, in Denmark and Great Britain $-40 \%$, in Japan and 
USA $-42 \%$, Canada $-56 \%$, Australia $-68 \%$, New Zealand $-72-77 \%$. Recently, in the scientific literature, reports have emerged about decrease in the sensitivity of representatives of Mycoplasmataceae family to preparations of fluoroquinolone group. Therefore, Zheng et al. (2021) revealed that out of 1,567 isolates of Ureaplasma urealyticum and Mycoplasma hominis, $18.8 \%$ of the cultures were resistant to ofloxacin, $14.7 \%$ to sparfloxacin, and only $7.8 \%$ to levofloxacin. In the article by Solís-Martínez et al. (2006), frequency of isolating M. hominis and U. urealyticum resistant to norfloxacin accounted for $67.8 \%$; and $57.6 \%$ were resistant to ofloxacin. The increase in the number of strains resistant to preparations of the fluoroquinolone group is attributed to emergence of mutations in genes responsible for synthesis of target-enzymes - bacterial topoisomerases. The basis for development of resistance to fluoroquinolones is amino acid replacements in the quinolone-binding pocket of sensitive enzymes, leading to decrease in their affinity to fluoroquinolones. Boujemaa et al. (2020) confirmed that 22 of 26 isolates of Ureaplasma isolated from the clinical material and resistant to ciprofloxacin had amino acid replacement (S83L) in one of subunits of topoisomerase IV - ParC.

Because the resistance of microorganisms to antibiotics over the recent years has achieved a threatening scale, it is specially important to make an annual assessment of dynamics of changes in the sensitivity of microorganisms based on continuous study of their properties in all etiologically significant strains isolated from clinical material. The conducted researches revealed increase in frequency of detection of strains of uropathogenic bacteria resistant to currently used antibiotic preparations occurring in 2019-2019 compared with the data of 2017: $10.3 \%$ and $6.4 \%$ increases in resistances representatives of the Mycoplasmataceae family to ciprofloxacin and ofloxacin respectively, $4.8 \%$ and $4.0 \%$ in Enterobacteriaceae to chloramphenicol and ampicillin respectively, and $8.9 \%$ in Staphylococcus genus to vancomycin. The data we obtained correlate with the results of the studies carried out by Patwardhan et al. (2017) and Saad et al. (2020).

Therefore, Patwardhan et al. (2017), during a study of the dynamics of annual changes (2009 to 2014) in the levels of resistance to antibiotics exerted by uropathogens isolated from patients at a hospital in North India, determined increase in the level of resistance of the bacterial cultures to norfloxacin from $30.0 \%$ to $41.4 \%$. Saad et al. (2020) demonstrated that for a seven-year period (2009-2015), in Sudan, the frequency of detection of strains of uropathogenic bacteria of Staphylococcus, Pseudomonas, Escherichia, Klebsiella, Proteus genera resistant to such antimicrobial drugs as amoxicillin/clavulanate, ampicillin/sulbactam, cefalexin, nitrofurantoin, co-trimoxazole, chloramphenicol, clindamycin, ciprofloxacin and norfloxacin increased from $2.0 \%$ (to ampicillin) to $15.0 \%$ (to cefalexin, clindamycin, chloramphenicol, norfloxacin). On the other hand, according to the data of $\mathrm{Li}$ et al. (2017), who analyzed the levels of resistance to antibiotics of uropathogens during 6 years in Southern China, Grampositive bacteria, including those of the Staphylococcus genus, were the most susceptible to linezolid and vancomycin, which contradicts the results we obtained. Such difference may be explained by geographic peculiarities of distribution of genes of resistance to antibiotics among microbial isolates. Moreover, there are certain differences in the schemes of prescriptions of antibacterial preparations in various countries, which may be also related to the differences in the parameters of resistance to antibiotics in various geographic regions. Thus, the recommended therapeutic scheme should take into account regional manifestation of resistance to particular drugs, observed in conditions of constant monitoring. Furthermore, for adequate therapy of diseases of the reproductive system, particularly, for effective selection of drugs, it is crucial to take into account possible polymicrobial pattern of diseases and have the possibility to simultaneously identify several pathogens. Those strategies allow one to use existing drugs during a longer period with better efficiency and prevent the manifestations of multi-resistant microbiota (Shaskolskiy et al., 2016).

The results obtained in the period of 2018-2019 indicate that in the urogenital system of the examined patients, strains of the uropathogenic microorganisms resistant to various antibiotics circulated with high frequency, and the levels of their resistance are tending to increase against drugs that are broadly used in therapy of infections and correction of dysbotic malfunctions.

\section{Conclusions}

The examination of patients of different sexes and age groups revealed the fact of dysbiotic disorders of the urogenital microbiome in $82.7 \%$ of patients - men and women of reproductive age.

We determined the dominant role of facultative anaerobes in the development of dysbiotic malfunctions caused by colonization by large amounts of conditionally pathogenic microorganisms: in women, Gardnerella was found in $86.1 \%$, Staphylococcus in $63.2 \%$, Streptococcus in $54.1 \%$, Candida in $69.3 \%$; in men, Streptococcus was found in $83.0 \%$, Staphylococcus in $79.4 \%$, Corynebacterium in $54.2 \%$ and Candida in $37.6 \%$ of the cases. Percentage share of obligate anaerobes was also quite high: from women there were isolated Prevotella - in 59.7\%, Peptostreptococcus - 53.2\%, Fusobacterium - 45.4\%; in men, Peptostreptococcus were isolated in $62.4 \%$, Clostridium - in 54.3\%, Bacteroides - in 32.5\% of the cases.

We determined high parameters of detection of antibiotic-resistant isolates of conditionally pathogenic microorganisms that circulate in the urogenital tract of patients with dysbiotic malfunctions belonging to the following families - Mycoplasmataceae $(78.6 \%)$, Enterobacteriaceae (56.0\%) - and genera - Staphylococcus (76.1\%), Gardnerella (24.3\%), Corynebacterium (21.3\%). The increase in the frequency of detection of resistant strains of uropathogenic bacteria to currently used antibiotic drugs for 2018-2019 compared with the data of 2017: 10.3\% and 6.4\% in representatives of the Mycoplasmataceae family resistant to ciprofloxacin and ofloxacin respectively, $4.8 \%$ and $4.0 \%$ in Enterobacteriaceae to chloramphenicol and ampicillin respectively, $8.9 \%$ in genus Staphylococcus to vancomycin.

\section{References}

Abelson, B., Sun, D., Que, L., Nebel, R., Baker, D., Popiel, P., Amundsen, C., Chai, T., Close, C., DiSanto, M., Fraser, M., Kielb, S., Kuchel, G., Mueller, E., Palmer, M., Parker-Autry, C., Wolfe, A., \& Damaser, M. (2018). Sex differences in lower urinary tract biology and physiology. Biology of Sex Differences, 9(1), 45.

Agar, A., Descalzi, B., Nally, M., Perez, D., \& Christiansen, P. (2020). Urogenital dysbiosis: The importance of a complete immune/nutritional evaluation and the therapeutic intervention with probiotics, vitamins and minerals. World Allergy Organization Journal, 13(8), 100319.

Aragón, I., Herrera-Imbroda, B., Queipo-Ortuño, M., Castillo, E., Del Moral, J., Gómez-Millán, J., Yucel, G., \& Lara, M. (2018). The Urinary tract microbiome in health and disease. European Urology Focus, 4(1), 128-138.

Bajic, P., Dombier, R., Doshi, C., Wolfe, A., Farooq, A., \& Bresler, L. (2019). Implications of the genitourinary microbiota in prostatic disease. Current Urology Reports, 20(7), 34.

Bitew, A., Abebaw, Y., Bekele, D., \& Mihret, A. (2017). Prevalence of bacterial vaginosis and associated risk factors among women complaining of genital tract infection. International Journal of Microbiology, 2017, 4919404.

Boboia, A., Florea, L. S., Turcu-Stiolica, A., Tăerel, A. E., Rais, C., Revnic, C., Florea, A., Vedeanu, N. S., Nastasă, C., \& Oniga, O. (2020). Decision analysis of antibiotic use. Farmacia, 68(4), 757-765.

Bondarenko, G., Dzhoraeva, S., Kondakova, A., Shcherbakova, I., Sobol, N., \& Ivantsova, H. (2017). Main agents of opportunistic infections of genitourinary tracts and their antibiotic resistance. Eureka: Health Sciences, 1(3), 18-25.

Boujemaa, S., Mlik, B., Allaya, A. B., Mardassi, H., \& Mardassi, B. B. A. (2020). Spread of multidrug resistance among Ureaplasma serovars, Tunisia. Antimicrobial Resistance and Infection Control, 9, 19.

Burnham, C. A. D., Leeds, J., Nordmann, P., O’Grady, J., \& Patel, J. (2017). Diagnosing antimicrobial resistance. Nature Reviews Microbiology, 15, 697-703.

Ceccarani, C., Foschi, C., Parolin, C., D’Antuono, A., Gaspari, V., Consolandi, C., Laghi, L., Camboni, T., Vitali, B., Severgnini, M., \& Marangoni, A. (2019). Diversity of vaginal microbiome and metabolome during genital infections. Scientific Reports, 9(1), 14095.

CLSI (2018). Methods for dilution antimicrobial susceptibility tests for bacteria that grow aerobically. 11th ed. CLSI standart M07. Clinical and Laboratory Standarts Institute, Wayne.

Conway, R., Cook, S., \& Soni, S. (2019). Antibiotic treatment of Mycoplasma genitalium infection. The Pharmaceutical Joumal, 303, 7928.

Drago, F., Ciccarese, G., Rebora, A., Cordara, V., \& Parodi, A. (2020). Common bacterial urogenital infections: A study on their aetiology and prevalence in a sexually transmitted infections centre. Joumal of the European Academy of Dermatology and Venerology, 34(10), e630-e632. 
Graspeuntner, S., Loeper, N., Künzel, S., Baines, J. F., \& Rupp, J. (2018). Selection of validated hypervariable regions is crucial in 16S-based microbiota studies of the female genital tract. Scientific Reports, 8(1), 9678.

Ho, H., Tan, M., Chen, M., Tan, T., Koo, S., Koong, A., Ng, L., Hu, P., Tan, K. Moey, P., Koh, E., Wong, C., Lye, D., \& Tan, N. (2019). Interaction between antibiotic resistance, resistance genes and treatment response for urinary tract infections in primary care. Journal of Clinical Microbiology, 57(9), e00143-19.

Kamińska, D., \& Gajecka, M. (2017). Is the role of human female reproductive tract microbiota underestimated? Beneficial Microbes, 8(3), 327-343.

Karou, S., Djigma, F., Sagna, T., Nadembega, C., Zeba, M., Kabre, A., Anani, K., Ouermi, D., Gnoula, C., Pietra, V., Pignatelli, S., \& Simpore, J. (2012). Antimicrobial resistance of abnormal vaginal discharges microorganisms in Ouagadougou, Burkina Faso. Asian Pacific Joumal of Tropical Biomedicine, 2(4), 294-297.

Knight, G. M., Davies, N. G., Colijn, C., Coll, F., Donker, T., Gifford, D. R., Glover, R. E., Jit, M., Klemm, E., Lehtinen, S., Lindsay, J. A., Lipsitch, M., Llewelyn, M. J., Mateus, A., Robotham, J. V., Sharland, M., Stekel, D., Yakob, L., \& Atkins, K. E. (2019). Mathematical modelling for antibiotic resistance control policy: Do we know enough? BMC Infectious Diseases, 19(1), 1011.

Koedooder, R., Mackens, S., Budding, A., Fares, D., Blockeel, C., Laven, J., \& Schoenmakers, S. (2019). Identification and evaluation of the microbiome in the female and male reproductive tracts. Human Reproduction Update, 25(3), 298-325.

Kudinova, A. G., Soina, V. S., Maksakova, S. A., \& Petrova, M. A. (2019). Basic antibiotic resistance of bacteria isolated from different biotopes. Microbiology, $88(6), 739-746$.

Kulchavenya, E. (2020). The best rules for antimicrobial stewardship in urogenital tract infections. Current Opinion in Urology, 30(6), 838-844.

Lee, K., Song, H., \& Kim, Y. (2020). The microbiome in urological diseases. Investigative and Clinical Urology, 61(4), 338-348.

Lewis, F., Bemstein, K., \& Aral, S. (2017). Vaginal microbiome and its relationship to behavior, sexual health, and sexually transmitted diseases. Obstetrics and Gynecology, 129(4), 643-654.

Li, J., Chiu, P., \& Ng, C. (2019). The impact of microbiome in urological diseases: A systematic review. International Urology and Nephrology, 51(10), 16771697.

Li, W., \& Ma, Z. (2019). Diversity scaling of human vaginal microbial communities. Zoological Research, 40(6), 587-594.

Li, X., Chen, Y., Gao, W., Ye, H., Shen, Z., Wen, Z., \& Wei, J. (2017). A 6-year study of complicated urinary tract infections in Southem China: Prevalence, antibiotic resistance, clinical and economic outcomes. Therapeutics and Clinical Risk Management, 13, 1479-1487.

Machowska, A., \& Lundborg, C. S. (2019). Drivers of irrational use of antibiotics in Europe. International Journal of Environmental Research and Public Health, $16(1), 27$.

MacIntyre, D., Sykes, L., \& Bennett, P. (2017). The human female urogenital microbiome: Complexity in normality. Emerging Topics in Life Sciences, 1(4), 363-372.

Magistro, G., \& Stief, C. (2019). The urinary tract microbiome: The answer to all our open questions? European Urology Focus, 5(1), 36-38.

Martin, D., \& Marrazzo, J. (2016). The vaginal microbiome: Current understanding and future directions. Journal of Infectious Diseases, 214(1), 36-41.

Mediati, D. G., Wu, S., Wu, W., \& Tree, J. J. (2021). Networks of resistance: Small RNA control of antibiotic resistance. Trends in Genetics, 37(1), 35-45.

Muhleisen, A., \& Herbst-Kralovetz, M. (2016). Menopause and the vaginal microbiome. Maturitas, 91, 42-50.

Neugent, M., Hulyalkar, N., Nguyen, V., Zimmern, P., \& De Nisco, N. (2020) Advances in understanding the human urinary microbiome and its potential role in urinary tract. American Society for Microbiology, 11(2), e00218-20.

Ortega Martell, J. (2020). Immunology of urinary tract infections. GMS Infectious Diseases, 8(21), 1-6.
Oztekin, C., Kaya-Sezginer, E., Yilmaz-Oral, D., \& Gur, S. (2018). Male urogenital disorders and metabolic syndrome: Possible links, characteristics and potential treatment strategies. Current Pharmaceutical Design, 24(9), 1019-1033.

Palchykov, V. A., Zazharskyi, V. V., Brygadyrenko, V. V., Davydenko, P. O. Kulishenko, O. M., Borovik, I. V., Chumak, V., Kryvaya, A., \& Boyko, O. O. (2019). Bactericidal, protistocidal, nematodicidal properties and chemical composition of ethanol extract of Punica granatum peel. Biosystems Diversity, 27(3), 300-306.

Patwardhan, V., Kumar, D., Goel, V., \& Singh, S. (2017). Changing prevalence and antibiotic drug resistance pattern of pathogens seen in community-acquired pediatric urinary tract infections at a tertiary care hospital of North India. Joumal of Laboratory Physicians, 9(4), 264-268.

Rigo, G. V., \& Tasca, T. (2020). Vaginitis: Review on drug resistance. Current Drug Targets, 21(16), 1672-1686.

Saad, D., Gameel, S., Ahmed, S., Basha, E., Osman, M., \& Khalil, E. (2020). Etiological agents of urinary tract infection and 7 years trend of antibiotic resistance of bacterial uropathogens in Sudan. The Open Microbiology Journal, 14, 312-320.

Schuppe, H., Pilatz, A., Hossain, H., Diemer, T., Wagenlehner, F., \& Weidner, W. (2017). Urogenital infection as a risk factor for male infertility. Deutsches Arzteblatt International, 114(19), 339-346.

Shaskolskiy, B., Dementieva, E., Leinsoo, A., Runina, A., Vorobyev, D., Plakhova X., Kubanov, A., Deryabin, D., \& Gryadunov, D. (2016). Drug resistance mechanisms in bacteria causing sexually transmitted diseases and associated with vaginosis. Frontiers in Microbiology, 7, 747.

Shoskes, D., Altemus, J., Polackwich, A., Tucky, B., Wang, H., \& Eng, C. (2016). The urinary microbiome differs significantly between patients with chronic prostatitis/chronic pelvic pain syndrome and controls as well as between patients with different clinical phenotypes. Urology, 92, 26-32.

Sirichoat, A., Lulitanond, A., Kanlaya, R., Tavichakomtrakool, R., Chanawong, A., Wongthong, S., \& Thongboonkerd, V. (2016). Phenotypic characteristics and comparative proteomics of Staphylococcus aureus strains with different vancomycin-resistance levels. Diagnostic Microbiology and Infectious Disease, $86(4), 340-344$.

Solís-Martínez, R., Vázquez-Castillo, T., Celis, S., \& Hernández-Callejas, L. (2006) Susceptibilidad de Mycoplasma hominis y Ureaplasma urealyticum ante diferentes antibióticos. Revista Medica de la Universidad Veracruzana, 6(2), 11-17.

Song, S., Acharya, K., Zhu, J., Deveney, C., Walther-Antonio, M., Tetel, M., \& Chia, N. (2020). Daily vaginal microbiota fluctuations associated with natural hormonal cycle, contraceptives, diet and exercise. MSphere, 5(4), e00593-20.

Stout, M., Wylie, T., Gula, H., Miller, A., \& Wylie, K. (2020). The microbiome of the human female reproductive tract. Current Opinion in Physiology, 13, 87-93.

Tang, J. (2017). Microbiome in the urinary system - a review. AIMS Microbiology, 3(2), 143-154.

Tran, K., \& Rybak, M. (2018). Beta-lactams combinations with vancomycin provide synergy against VSSA, hVISA, and VISA. Antimicrobial Agents and Chemotherapy, 62(6), e00157.

Valentine-King, M., \& Brown, M. (2017). Antibacterial resistance in Ureaplasma species and Mycoplasma hominis isolates from urine cultures in college-aged females. Antimicrobial Agents and Chemotherapy, 61(10), e01104-17.

Wagenlehner, F. (2020). Urogenital infections. World Joumal of Urology, 38(1), 1-2.

Xu, J., Bian, G., Zheng, M., Lu, G., Chan, W., Li, W., \& Du, Y. (2020). Fertility factors affect the vaginal microbiome in women of reproductive age. American Joumal of Reproductive Immunology, 83(4), e13220.

Zazharskyi, V. V., Davydenko, P. O., Kulishenko, O. M., Borovik, I. V., \& Brygadyrenko, V. V. (2019). Antimicrobial activity of 50 plant extracts. Biosystems Diversity, 27(2), 163-169.

Zazharskyi, V. V., Davydenko, P. O., Kulishenko, O. M., Borovik, I. V., Zazharska, N. M., \& Brygadyrenko, V. V. (2020). Antibacterial and fungicidal activities of ethanol extracts of 38 species of plants. Biosystems Diversity, 28(3), 281-289.

Zheng, W., Zhang, W., Cui, D., Nie, Z., Ding, B., Cheng, J., \& Mei, C. (2021). Examination of Ureaplasma urealyticum and Mycoplasma hominis in 4082 Chinese patients. Brazilian Journal of Medical and Biological Research, 54(2), e10099. 